\title{
Lavender aromatherapy: a systematic review from essential oil extraction and administration to cognitive enhancing effects.
}

\author{
Eleonora Malloggi ${ }^{1 \dagger}$, Danilo Menicucci ${ }^{1+*}$, Valentina Cesari ${ }^{1}$, Sergio Frumento ${ }^{1}$, Angelo Gemignani ${ }^{1}$, Alessandra \\ Bertoli $^{2}$
}

tauthors contributed equally to this manuscript

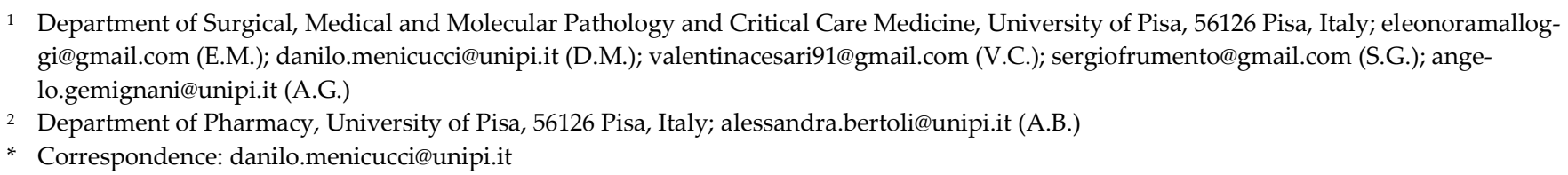

\begin{abstract}
Modern society is reviving the practice of aromatherapy, and lavender is reported to be the most worldwide purchased plant for essential oils (EO) extraction. Since odors can modulate cognitive functions acting through specific neuroanatomical pathways, lavender EO inhalation can enhance cognition. Taking into account EO quality and diffusion devices, we conducted a systematic review on the effects of lavender EO inhalation on arousal, attention and memory in healthy subjects. Starting from this new multidisciplinary perspective, cognitive effects were revised to link outcomes to effective and reproducible aromatherapy protocols. A systematic search on MEDLINE database using Cognitive Atlas and plant authenticity-related keywords was performed. Among the 806 articles yielded, 11 articles met eligibility criteria. Subjects administered with lavender EO displayed arousal decrease and sustained attention increase. Puzzling results were obtained regarding memory. Lack of EO quality assessment and high heterogeneity in inhalation protocols did not allow assessing whether different EO composition differently modulates cognition and whether placebo or expectancy effect can be discerned from EO effect itself. However, GABAergic pathway modulation exerted by linalool, a major lavender EO constituent, explains arousal reduction and sustained attention enhancement. In conclusion, aromatherapy can be an innovative, practical and non-invasive tool to prevent cognitive lapses.
\end{abstract}

Keywords: aromatherapy; essential oil; lavender; cognitive functions; arousal; attention; memory

\section{Introduction}

Over the last few decades, the traditional medical practice of aromatherapy, defined as "the controlled use of plant essences for therapeutic purposes" [1,2], has been revived by scientific literature and has risen to the role of the most common complementary medicine.

Indeed, the International Federation of Essential Oils and Aroma Trades (IFEAT) reported a rapid growth in aromatic plants cultivation and lavender as the most worldwide purchased raw material for essential oils (EO) extraction [3]. Actually, since the discovery of its soothing effects in the early 1900s [4], lavender aromatherapy has widely spread among alternative or complementary usages, ranging from antiemetic to soothing and pain treatment up to lately discovered sedative and cognitive effects [5].

EO and their derivatives are constituted by many different natural components which are strictly related to raw plant origin and transformation process. Therefore, the preliminary quality control of the tested EO is a crucial step to guarantee reproducible and safe aromatherapy protocols. In this context, EO are monitored by scientific committees of different international institutions to ensure phytochemical quality and customers' safety [6-11]. In particular, the International Standard Organization (ISO) established specific standards for extraction and analytical composition in order to define EO quality grades [12-14] and in 2012 the European Medicines Agency (EMA) Committee on Herbal Medicinal Products (HMPC) adopted a community herbal monograph on Lavandula angustifolia Miller aetheroleum, providing specific posologies and oral administration methods. The specific HMPC assessment concluded that lav- 
ender oil showed a possible effect on several clinical conditions, asserting that its use in the medical field can be based on its long-standing usage.

Since odor inhalation can modulate cognitive functions through specific neuroanatomical pathways triggered by olfactory bulb, an increasing body of scientific evidence has recently been focusing on the effects of lavender EO inhalation on cognitive functions [15]. It has been stated the role of olfactory system's anatomical and functional connectivity with subcortical hubs, suggesting an indirect role of olfactory stimulation in arousal, reward learning, emotions and memory [16]. Furthermore, the high degree of functional connectivity between olfactory systems and neocortex suggests that olfactory stimulation may play an important role in the modulation of higher cognitive functions [17].

In light of the foregoing neuroanatomical evidence, a systematic and integrated study on the impact of lavender EO inhalation on cognitive functions is needed, since, due to its ascertained soporific effects, lavender properties on cognition enhancement have been scarcely investigated and remain controversial. Furthermore, most studies focused on compartmentalized outcomes ranging from behavioral and physiological measures to phytochemical composition of EO. As regards lavender EO composition, linalool and linalyl acetate are the two main components whose mode of action has been demonstrated responsible for psychophysiological effects [18]. Based on these premises, a systematic characterization of the effects of lavender EO on cognition from a multidisciplinary perspective would help in the comprehension of lavender EO inhalation effects on cognitive enhancement. In this context, different types of EO diffusion devices may also have an impact on administration procedure, since EO quality can be differently affected by the device employed [19]. Thus, taking into account the EO phytochemical parameters and administration methods, the aim of this systematic review is to assess in healthy subjects whether different lavender EO compositions can differently influence core cold cognitive functions such as vigilance and arousal, attention and memory or whether these alleged effects hold true regardless of predetermined EO composition.

\section{Materials and Methods}

This systematic review was carried out according to the Preferred Reporting Items for Systematic Reviews and Meta-Analyses (PRISMA) guidelines, which comprises a checklist to ensure the quality of systematic reviews [20], reported in Supplementary Table 1 (S1). The protocol for the present systematic review has been submitted for registration (ID number 180709) to PROSPERO international prospective register for systematic reviews database [21].

To develop an effective search strategy, we adopted the Population, Intervention, Comparison, Outcomes and Study design (PICOS) strategy [22], reported in Supplementary Table 2 (S2). The literature search was conducted in March 2021 and comprised three phases: 1) Identification: a systematic search based on queries on the electronic database MEDLINE of articles from 2000 to present; 2) Screening: a manual screening of the articles yielded in phase 1) by evaluating title and abstract with the eligibility criteria illustrated in Supplementary Table 3 (S3); 3) Final eligibility: a more in-depth assessment of the remaining papers on the basis of the full-text. At phase 1, boolean operators "AND" and "OR" were applied to combine a list of keywords related to lavender and a list of keywords related to cognitive functions retrieved from Cognitive Atlas website [23], reported in Supplementary Table 4 (S4). At phases 2 and 3, eligibility criteria were applied and only studies investigating cognitive effects of lavender EO inhalation in healthy subjects were retained.

Supplementary Table 4 (S4) summarizes the search steps performed on the database. Articles retrieved from MEDLINE were merged into the Mendeley database [24] and duplicates were automatically removed using the desktop Mendeley reference manager. During the inclusion phase, articles containing words related to agricultural and botanical fields and to medical treatments were excluded; to this aim, a list of excluding words related to the aforementioned fields was used to automatically exclude these studies (list of excluding words is reported in Table S5).

To assess the quality of evidence of the studies, we used the Newcastle-Ottawa Scale (NOS, Table S6) for the evaluation of randomized-controlled studies [25]. The scale scores the quality of reporting with a maximum of 9 stars for higher quality. According to NOS, each study is judged for: selection of the study groups; comparability of the groups; and ascertainment of either the exposure or outcome of interest. In this process, two independent reviewers (E.M. and V.C.) scored each study according to NOS criteria. Any disagreements were resolved through consensus of all authors. 


\section{Results}

Our search yielded 806 articles. After duplicates removal and screening phase, 31 full-text articles were retained according to the eligibility criteria. Twenty of them were excluded for the following reasons: 14 studies included mixed EO; one study did not investigate cognitive functions; one study used lavender EO inhalation as a control condition; 2 studies used lavender EO topical application; 2 studies were based on a within-group without randomized controlled design. Articles selection process is illustrated in Figure 1. After the inclusion phase, articles were divided into three macro-categories regarding psychological macro-domains, by evaluating the studies' outcomes: arousal, attention and memory. Detailed information is provided in Table 1. Finally, 11 articles were revised. NOS scores were adequate for all studies and ranged from 5 to 7 . According to Table 1, all studies recruited young adults and adults, except for two studies [26,27] in which elderly subjects were recruited too. No age effect was observed. Furthermore, no effects elicited by different $\mathrm{EO}$ administration timing were observed.

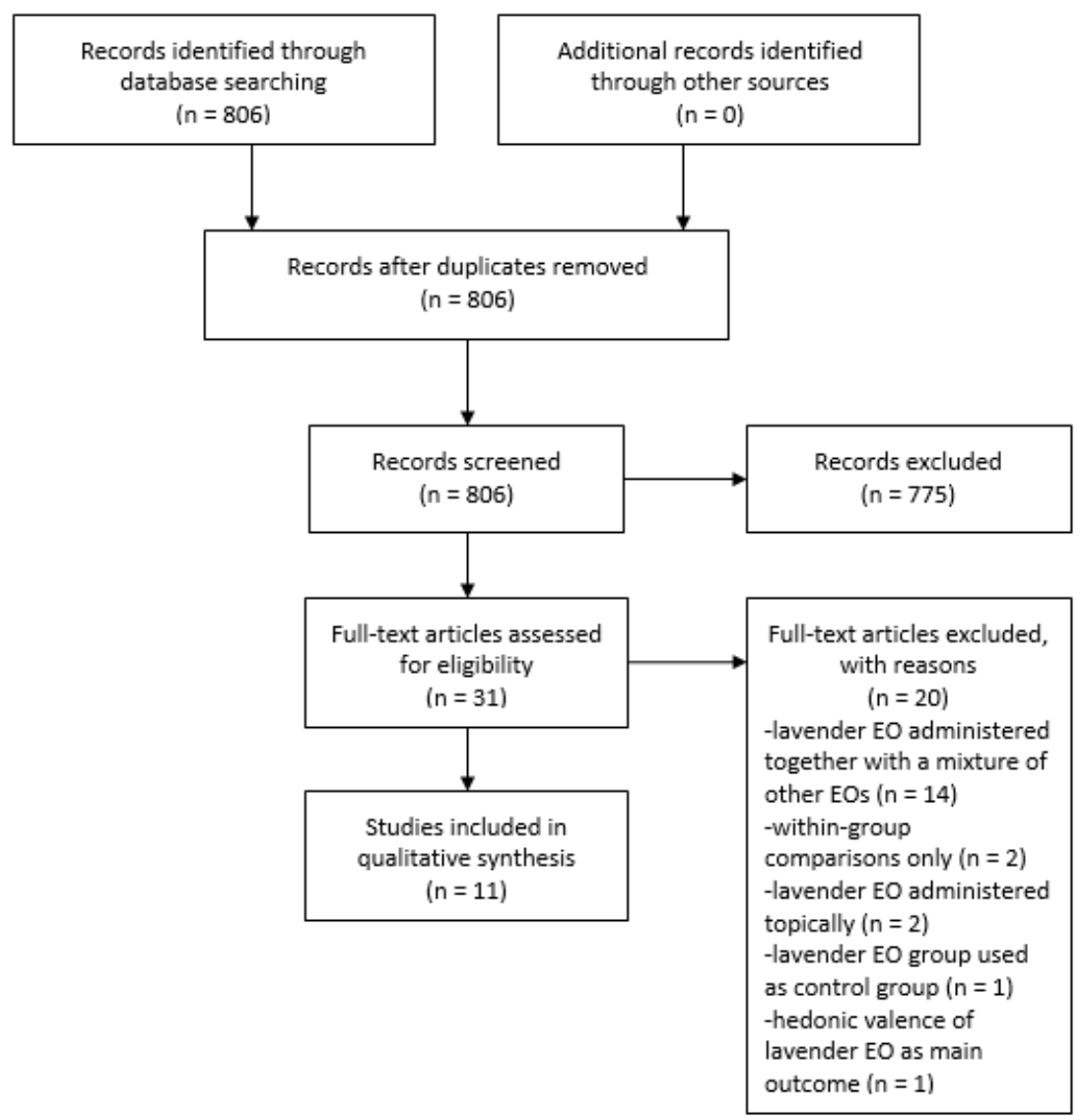

Figure 1. Flow chart of the selected studies. 
Table 1. Cognitive and phytochemical primary outcomes

\begin{tabular}{|c|c|c|c|c|c|c|c|c|c|c|c|c|}
\hline Study & Design & $\begin{array}{l}\text { Subjects } \\
\text { number }\end{array}$ & Age & Groups & Procedure & Measurement & Results & $P$ value & Odor awareness & $\mathrm{EO}^{2}$ characteristics & $\mathrm{EO}^{2}$ quali-quantitative analyses & Diffusion system \\
\hline \multicolumn{13}{|c|}{ AROUSAL } \\
\hline \multirow{7}{*}{ Kiecolt-Glaser et al., 2008} & \multirow{7}{*}{$\begin{array}{l}\text { Single blind/primed, } \\
\text { crossover }\end{array}$} & \multirow{7}{*}{18} & \multirow{7}{*}{$\begin{array}{l}\text { Young } \\
\text { adults, } \\
\text { adults }\end{array}$} & \multirow{7}{*}{$\begin{array}{l}\text { Lavender, lemon, } \\
\text { control (blind and } \\
\text { primed subgroups } \\
\text { for each group) }\end{array}$} & \multirow{7}{*}{$\begin{array}{c}\text { Physiological } \\
\text { parameters were } \\
\text { assessed before } \\
\text { and after subjects } \\
\text { undergoing cold } \\
\text { pressor test while } \\
\text { inhaling } \mathrm{EO}^{2}\end{array}$} & Norepinephrine & Arousal decrease & $P=0,019$ vs lemon & \multirow{7}{*}{ Aware } & \multirow{7}{*}{$\mathrm{PVE}^{12}$} & \multirow{7}{*}{ GC-MS'17 } & \multirow{7}{*}{$\mathrm{c}^{18}$} \\
\hline & & & & & & $\mathrm{BP}^{4}$ & No changes & Not significant & & & & \\
\hline & & & & & & $\mathrm{HR}^{5}$ & \begin{tabular}{|c|} 
Arousal increase \\
in primed \\
groups only
\end{tabular} & Not significant & & & & \\
\hline & & & & & & Salivary cortisol & No changes & $\begin{array}{c}\mathrm{P}=0,002 \text { blind vs } \\
\text { not blind }\end{array}$ & & & & \\
\hline & & & & & & $\begin{array}{c}\text { Blastogenesis response: } \\
\text { lymphocyte response to } \\
\text { mitogens }\end{array}$ & Arousal decrease & $\begin{array}{l}-\mathrm{P}=0,001 \text { vs water } \\
-\mathrm{P}=0,009 \text { vs lemon } \\
\text { in primed groups }\end{array}$ & & & & \\
\hline & & & & & & Interleukin- 6 and -10 & No changes & Not significant & & & & \\
\hline & & & & & & $\begin{array}{c}\text { Delayed hypersensitivity to } \\
\text { candida }\end{array}$ & Arousal decrease & $\mathrm{P}=0,02$ vs water & & & & \\
\hline
\end{tabular}




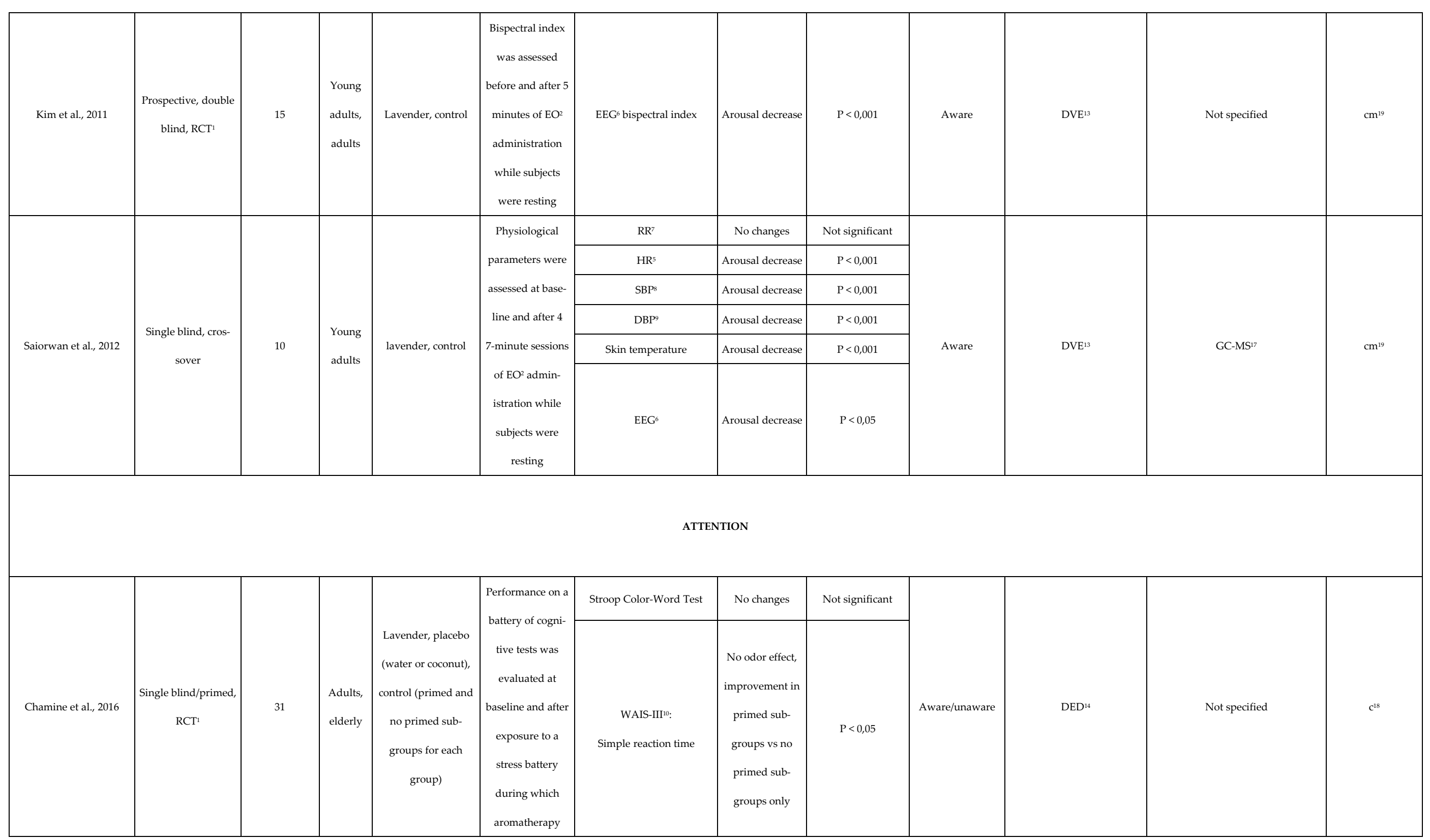




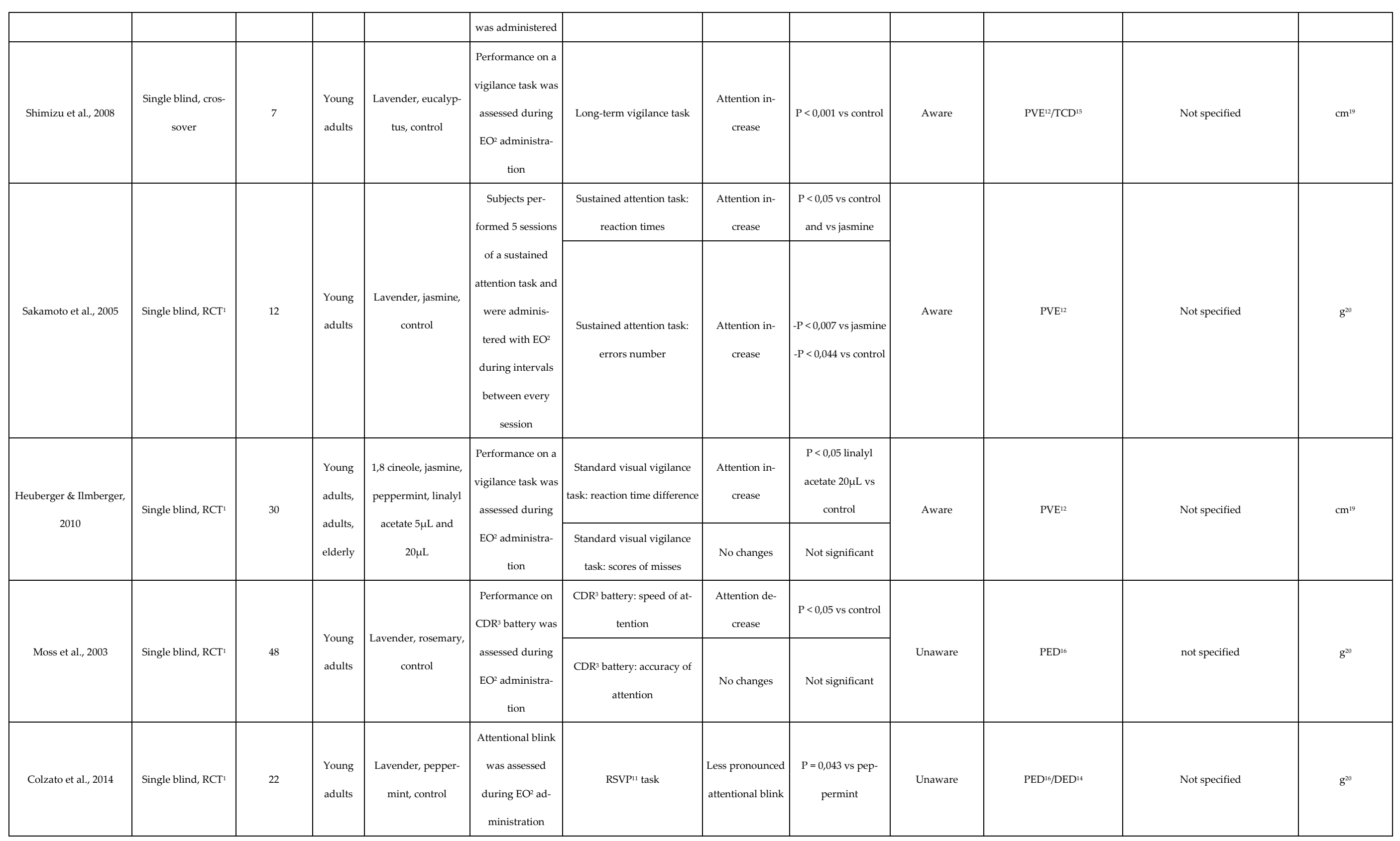




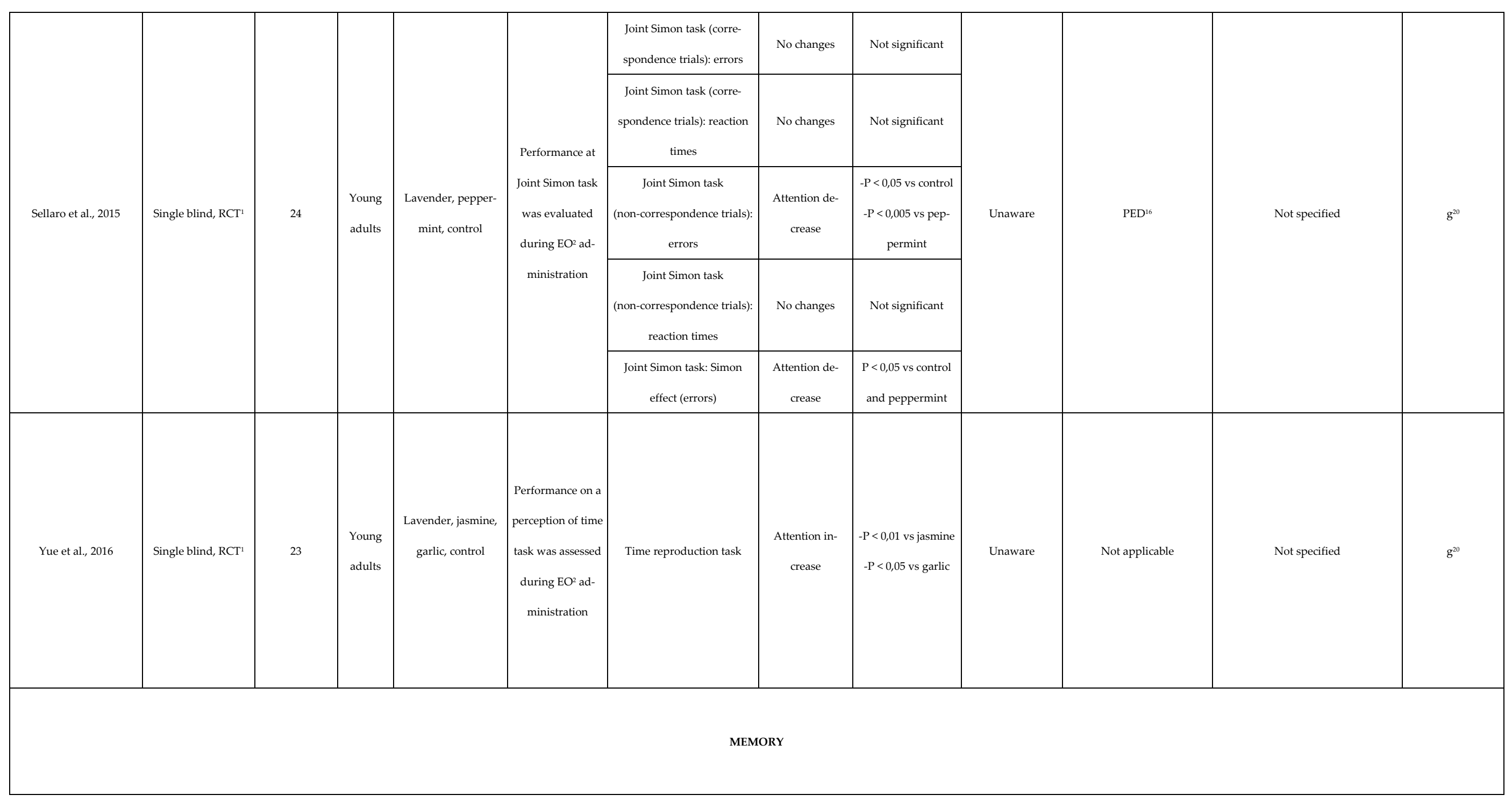




\begin{tabular}{|c|c|c|c|c|c|c|c|c|c|c|c|c|}
\hline Chamine et al., 2016 & $\begin{array}{c}\text { Single blind/primed, } \\
\mathrm{RCT}^{1}\end{array}$ & 31 & $\begin{array}{l}\text { Adults, } \\
\text { elderly }\end{array}$ & $\begin{array}{l}\text { Lavender, placebo } \\
\text { (water or coconut), } \\
\text { control (primed and } \\
\text { no primed sub- } \\
\text { groups for each } \\
\text { group) }\end{array}$ & \begin{tabular}{|c} 
Performance on a \\
battery of cogni- \\
tive tests was \\
evaluated at \\
baseline and after \\
exposure to a \\
stress battery \\
during which \\
aromatherapy \\
was administered
\end{tabular} & $\begin{array}{l}\text { WAIS-III'vi: digit span back- } \\
\qquad \text { ward }\end{array}$ & $\begin{array}{c}\text { Memory impro- } \\
\text { vement }\end{array}$ & $\begin{array}{c}-\mathrm{P}=0,008 \text { vs coco- } \\
\text { nut } \\
-\mathrm{P}=0,021 \text { vs water }\end{array}$ & Aware/unaware & DED" & Not specified & $\mathrm{c}^{18}$ \\
\hline Moss et al., 2003 & Single blind, RCT' & 48 & $\begin{array}{l}\text { Young } \\
\text { adults }\end{array}$ & $\begin{array}{c}\text { Lavender, rosemary, } \\
\text { control }\end{array}$ & $\begin{array}{c}\text { Performance on } \\
\mathrm{CDR}^{3} \text { battery was } \\
\text { assessed during } \\
\mathrm{EO}^{2} \text { administra- } \\
\text { tion }\end{array}$ & $\mathrm{CDR}^{3}$ battery & Memory decline & $\begin{array}{c}-\mathrm{P}<0,05 \text { vs rose- } \\
\text { mary } \\
-\mathrm{P}<0,01 \text { vs control }\end{array}$ & Unaware & PED'6 & Not specified & $\mathrm{g}^{20}$ \\
\hline
\end{tabular}


${ }^{1} R C T$, randomized-controlled trial; ${ }^{2} E O$, essential oil; ${ }^{3} C D R$, cognitive drug research; ${ }^{4} B P$, blood pressure; ${ }^{5} H R$, heart rate; ${ }^{6} E E G$, electroencephalogram; ${ }^{7} R R$, respiratory rate; ${ }^{8} S B P$, systolic blood pressure; ${ }^{9} \mathrm{DBP}$, diastolic blood pressure; ${ }^{10} \mathrm{WAIS}$-III, Wechsler Adult Intelligence Scale-III; ${ }^{11}$ RSVP, rapid serial visual presentation; ${ }^{12} P V E$, pure volatile essential oil; ${ }^{13} D V E$, diluted volatile essential oil; ${ }^{14} D E D$, diluted essential oil drops; 15 TCD, target compound diffusion; ${ }^{16}$ PED, pure essential oil drops; ${ }^{17}$ GC-MS, gas-chromatography mass-spectrometry; ${ }^{18} \mathrm{c}$, cotton pad/gauze/linen fabric (two layers under nose, direct diffusion system); ${ }^{19} \mathrm{~cm}$, cotton pad/gauze/linen fabric/ball in surgical mask and oxygen flow/inhalers (direct diffusion system); ${ }^{20} \mathrm{~g}$, candle diffuser/soaked pad under bench/tank-becker (no electrical diffuser, indirect diffusion system).

\subsection{Phytochemical and diffusion parameters}

In the revision process, the selected articles were revised by taking into account four key points of EO selection and aroma diffusion: (i) quality parameters reported either in commercial documentation or lab-scale research data, (ii) sample preparation procedures, (iii) diffusion devices and (iv) subjects awareness towards administration procedure depending on the device used. Selection process is illustrated in Figure 2.

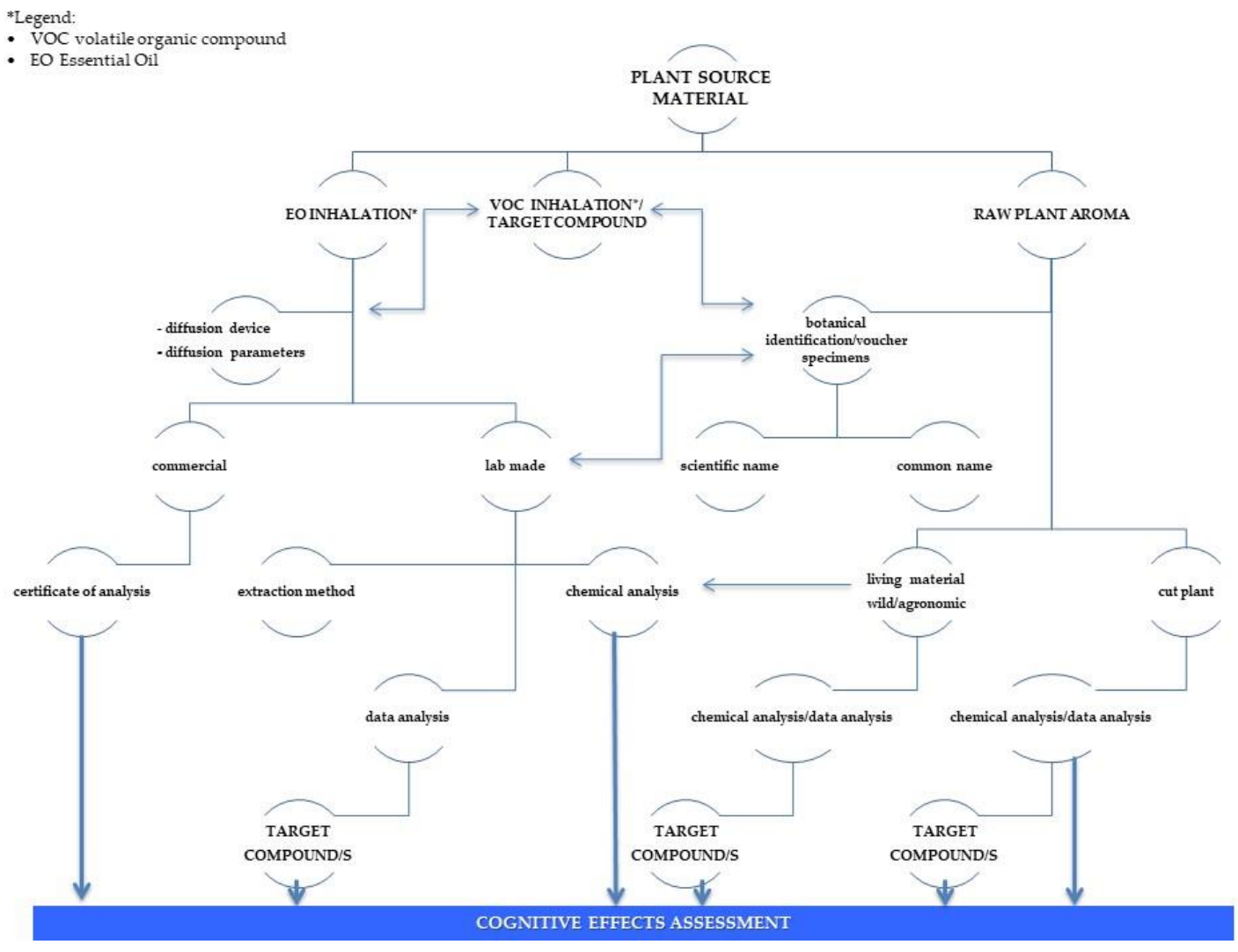

Figure 2. Quality assessment for inhaled sample. Flow diagram illustrating EO quality and administration systems selection as a preliminary step leading to cognitive effects assessment.

Quality parameters and sample preparation procedures were not always reported in the selected studies [28-30]. Different aromatic products were used by Heuberger \& Ilmberger [27] such as commercial singular volatile compounds, 
EO and absolute ether extract, which are defined with the general term "fragrances". The authors did not specify either if the EO were synthetic or isolated from plant material nor the quality analytical results of EO purity and composition. Although Kiecolt-Glaser et al. [31] did not report compositional data, theirs was the only experimental procedure based on lavender EO monitoring in order to avoid compositional variability. In Saiorwan and colleagues' study [32], the quality control of commercial EO lavender was carried out by gas chromatography-mass spectrometry analysis, but only the relative percentage of linalool $(31.9 \%)$ and linalyl acetate $(32.5 \%)$ were shown. Despite these two biomarkers being detected in the same amount, the authors considered only linalool as the main compound whose effects on cognitive functions were studied. In other cases, the scientific botanical name of the lavender raw material was the only certified information and the EO composition was not reported at all [26,30]. Shimizu and colleagues [33] reported no information about linalyl acetate level in the tested EO sample although the study considered the singular EO compound linalyl acetate as the main active lavender constituent.

Regarding diffusion devices, a variety of facilities with different diffusion methods have been described among the studies. According to EO administration method, two types of devices were identified: 1) direct administration (face systems) including steam diffusion, mask, natural volatile system lab-scale, cotton pad under nose; 2) indirect administration (environmental systems) including cotton pad under seat, candle, electric devices. Direct administration methods allowed subjects to be aware of the procedure, while indirect administration methods prevented subjects awareness towards protocol (see Table 1).

Six studies employed direct EO administration systems [26,27,31-34]. Shimizu and colleagues [33] used a lab-scale Natural Volatile Delivery System to spread lavender EO and linalyl acetate in the room environment, avoiding dishomogeneous EO dispersion. This device was placed 10 centimeters below the nose of each subject and allowed EO flow at constant pressure and quantity (amount per liter of air). Heuberger \& Ilmberger [27], Saiorwan et al. [32] and Kim et al. [34] administered EO by means of inhalation from a face mask. In particular, Heuberger \& Ilmberger [27] applied to subjects a surgical mask coated with lavender EO; Saiorwan and colleagues [32] mixed volatile components of EO with oxygen through a pump connected to an oxygen mask; Kim and colleagues [34] applied a cotton swab soaked with EO to an oxygen mask. No indication about the operating oxygen mask flow has been described. Chamine et al. [26] and Kiecolt-Glaser et al. [31] used tissue diffusion method by applying a cotton swab below the subjects' nose. In their study, Kiecolt-Glaser et al. [31] used a commercial lavender EO, identified by its specific tradename and producer, diluted in water (four drops/ $30 \mathrm{ml}$ ).

Five studies used indirect EO administration systems [28-30,35,36]. Sakamoto et al. [35] and Yue et al. [36] used general evaporative methods to diffuse a lavender and jasmine EOs samples. Authors also described the room parameters to guarantee insulation conditions and homogenous EO concentration in the room. A testing aromatic delimited room was also used also by Moss and colleagues [28], who employed a dedicated cubicle for a more controlled scent room applying a soaked cotton pad in a container under the subject's seat. Colzato et al. [29] and Sellaro et al. [30] administered EOs by means of a candle for a fixed exposure time.

\subsection{Effects of lavender EOs inhalation on arousal}

Three studies investigated lavender aromatherapy effects on arousal in healthy subjects [31,32,34]. Physiological measurements were assessed at baseline and after exposure to EO. In Saiorwan and colleagues [32] and in Kim and colleagues [34] studies, participants inhaled EO while resting, whereas, in Kiecolt-Glaser and colleagues [31] study, participants underwent a test that induces a stress response.

Kiecolt-Glaser and colleagues [31] found complex results while studying physiological responses to the cold pressor test as a function of inhaled EO while manipulating odor expectancy (priming). In their experiment they found that norepinephrine levels following the cold pressor test diminished in water and lavender conditions compared to lemon condition; delayed hypersensitivity to candida, an index of T-cells immunity, and blastogenesis response to mitogens, that are markers of stress-arousing response, were smaller after lavender condition compared to water condition.

Primed and blind groups did not differ. Blastogenesis response to mitogens was higher in lavender primed group than in water and lemon primed groups. EO did not alter the following stress-related parameters: Interleukin-6 and Interleukin-10 levels, salivary cortisol, heart rate (HR) or blood pressure (BP); however, expectancy effect was revealed for 
HR, since primed groups showed higher HR increase after cold pressor test, but also larger HR decrease during recovery as compared to blind groups.

Saiorwan and colleagues [32] assessed central and peripheral correlates of arousal level. EEG measures revealed widespread increase of power in alpha and theta bands, while autonomic parameters showed reduction of HR, systolic and diastolic BP and skin temperature in the lavender group compared to the control group (administered with base oil), but no differences were shown in respiratory rate.

Kim and colleagues [34] assessed arousal with the bispectral index (BIS), an electroencephalogram derivative index which reflects the level of consciousness during sedation and acupuncture [37,38], while inserting a needle into the forearm skin to induce a stress-arousing state in healthy participants. Authors found a decrease of arousal in the lavender EO group compared to the control group, whose participants were administered with oxygen only.

\subsection{Effects of lavender EO inhalation on attention}

Eight studies investigated the effects of lavender EO on attention in healthy subjects. All studies assessed performance on cognitive tasks while subjects were inhaling EO, except for Sakamoto and colleagues [35] study, in which participants were administered with EO during intervals between cognitive task sessions.

Chamine and colleagues [26] implemented a mixed design in which lavender and coconut groups were divided into two subgroups: one group was told it would receive a relaxing aroma, the other one was told that the aroma would have relaxing effects or not. Control group was administered with water and received no priming. Participants then underwent a stress battery including emotional and physical stressors while inhaling lavender or coconut EO or distilled water. Authors conducted cognitive and physiological assessment to detect the effect of EO controlling for expectation. Authors did not find significant changes after EO inhalation for Stroop Color-Word test [39] (a task in which the colour of the ink of a list of colours names does not match with the semantic significance of the words and the subjects are required to say the name of the ink, instead of reading the colour name) and WAIS-III Letter-Number Sequencing [40] (a task in which the participants must first say the numbers in ascending order and then the letters in alphabetical order), but faster reaction times were detected among primed subgroups, regardless of the EO. Physiological assessment showed changes in cortisol level and in respiratory rate, but chromogranin A level, a reliable marker of psychological stress, was found to be lower in the control group as compared to both EO groups.

Shimizu and colleagues [33] reported faster reaction times during a sustained attention task after lavender EO administration, compared to the control group that received no odor.

In Sakamoto and colleagues' study [35] a sustained attention task was performed by participants, showing that, compared to jasmine and no-odor groups, lavender EO produced faster reaction times and improved accuracy during all task sessions.

Heuberger and Ilmberger's study [27] showed faster reaction times during a vigilance task in participants who had inhaled $20 \mu \mathrm{L}$ of linalyl acetate as compared to those in the control group who were administered with water. This result was not significant for $5 \mu \mathrm{L}$ of linalyl acetate. Reaction times decrease was correlated with subjective ratings of fragrance valence: the more pleasant the lavender fragrance was rated, the faster were the reaction times. Few errors were correlated with subjective ratings too: the more stimulating the water was rated, the fewer were the errors.

On the contrary, Moss and colleagues [28] reported slower reaction times induced by lavender inhalation during a vigilance task, compared to rosemary and no-odor groups.

Colzato and colleagues [29] investigated the effects of lavender and peppermint EOs inhalation on the attentional blink, that reflects temporal limitations in visual attention allocation, assessed with a RSVP task [41], a task in which subjects are required to detect a target stimulus embedded in a stream of visual stimuli. Attentional blink was found to be less pronounced among participants in the lavender group as compared to no-aroma group, indicating a less focused attentional state induced by lavender. 
Sellaro and colleagues [30] investigated Simon effect [42] in participants inhaling lavender and peppermint EOs and in participants exposed to no aroma. This effect is the difference in accuracy or reaction time between trials in which stimulus and response are on the same side and trials in which they are on opposite sides, with responses being generally slower and less accurate when the stimulus and response are on opposite sides. More errors in the stimulus/response locations discordance condition were found in the lavender group compared to peppermint or control groups.

Yue and colleagues [36] investigated how time perception varied depending on different EO inhalation, as a measure of odor-dependent attentional deployment. The authors found a longer reproduction of time intervals than the actual time durations in the lavender group, compared to garlic and jasmine groups.

\subsection{Effects of lavender EO inhalation on memory}

Two studies investigated lavender EOs effects on memory in healthy subjects, reporting different results. Performance on cognitive tasks was assessed during EO administration.

Chamine and colleagues [26] found working memory performance improvement after lavender inhalation as compared to placebo and water inhalation; memory was assessed by Wechsler Adult Intelligence Scale-III (WAIS-III) digit span backward [40]. Neither priming nor placebo effects were reported. However, Moss and colleagues [28] showed that participants who had inhaled lavender EO had worse working memory performance as compared to the rosemary group or the control group that received no odor. Working memory was assessed with Cognitive Drug Research battery [43] which comprises numerical working memory and spatial working memory; for both tasks, speed and accuracy were measured.

\section{Discussion}

This systematic review aims to provide a comprehensive qualitative synthesis of psychophysiological outcomes of lavender EO inhalation, taking into consideration its quality issues as well as diffusion systems.

\subsection{EO quality control and trial intervention}

Due to the EO compositional complexity, its chemical profile should be considered a preliminary crucial step to assure research quality and safety in aromatherapy.

Despite the importance of providing the gas chromatography-mass spectrometry composition, lot number and trade name of EO sample, in most of the revised papers it was not possible to understand the EO quality of the inhaled sample. In a few cases [27,32,33], just two main major constituents, linalool and linalyl acetate, have been described. However, the presence of other typical minor compounds has not been provided. Monitoring the EO compositional data is essential in order to avoid adverse effects. For this reason, even when the commercial linalyl acetate or other singular chemical standard is used, compositional data should be reported to guarantee purity issues and to exclude the presence of hazard or allergenic additives [11].

Considering lavender EO composition complexity and variability, quali-quantitative characterization is necessary to determine the contribution of each component. Focusing on the main lavender constituents, the ratio between linalool and linalyl acetate in the EO lot sample should be stated, as linalyl acetate derives from linalool during the extraction process. Furthermore, the monoterpene linalool (3,7-dimethylocta-1,6-dien-3-ol) has been generally reported to influence the transmission of odor stimulation. In fact, the oxygen atom in its structure allows hydrogen bond formation with a hydrogen atom on the olfactory receptor. In addition, the chemical relationship between linalyl acetate and linalool is really tight because linalool is also the common and direct product of linalyl acetate hydrolysis together with acetic acid. Therefore, lavender EO activity could be strongly influenced by the linalyl acetate hydrolysis in favor of linalool levels followed by the unpleasant pungent odor of acetic acid [44]. 
Among the revised papers, Kiecolt-Glaser et al. [31] used a lavender EO extracted by steam distillation in lab-scale and analysed it before the intervention by gas chromatography-mass spectrometry. They monitored lavender EO also during the successive experimental sessions in order to avoid any compositional variability, but without reporting compositional data. Colzato and collaborators [29] considered linalool as the main component of lavender EO and, most importantly, they suggested its role in attention increase: in this context, the authors only measured linalyl acetate as reference control. Both linalool and linalyl acetate are reported to have complementary mechanisms which seem to be responsible for lavender EO cognitive effects $[45,46]$. On the other hand, other studies reported only linalool to be responsible for nervous system modulation. Regarding the qualification of linalyl acetate as character impact compound reported in Heuberger \& Ilmberger [27], Lavandula angustifolia Miller EO shows quite similar amounts of both linalyl acetate and linalool (ranges 7-56, 6-50 respectively; generally (30-35\%), following Pharmacopoeia [6] or ISO/AFNOR guidelines [11]. Therefore, both these compounds have been believed to be active substances. Nevertheless, Heuberger \& Ilmberger [27] asserted linalyl acetate to be the main psychotropic active principle. In light of the linalool and linalyl acetate structure-activity relationship, the EO profile in toto has to be considered due to the fact that minor constituents can play a crucial role in a specific olfactory stimulation, too. In addition, the authors used essential oils and an absolute ether extract introducing all of them by the general terms "fragrances". They specified the related provider, but no mention if they are synthetic or isolated from plant material or the quality control results of these materials in terms of their purity for the substances and composition for EO.

Another important drawback is the wide variety of vehicles used to dilute the inhaled sample, since they could interfere with the lavender EO olfactory stimulation. In this context, Diego et al. [47] diluted and administered lavender EO using grapeseed oil (10\%), while Saiorwan and collaborators [32] confirmed the relaxing lavender EO effects by dilution in sweet almond oil (10\%) or jojoba oil (2\%) in a previous work [34].

To ensure homogeneous experimental procedures and treatment, the established and controlled exposition time should be associated with dimensioned room, air ventilation parameters and administration device, in order to allow protocol reproducibility and results comparability.

As regards diffusion systems, indirect administration represents a practical and cheap method that allows blindness toward procedure; however, while the oil's volatile components are gradually released into the air, aroma quick dissipation may cause chemical degradation of some natural lavender constituents [19]. For example, circumscribed steam diffusion, used by Sakamoto and colleagues [35] (a tank with boiled water), allows a quick release of aroma into the room, but it does not guarantee a long-lasting and constant permanence of aroma in the air, since the use of heat may invalidate EO quality by disrupting its constituents [19]. Furthermore, in order to guarantee a sufficient amount of aroma for a proper temporal range into the room, the tank used in the study was not small enough to ensure subject blindness toward procedure, so, in this case, indirect administration method did not guarantee subjects unawareness towards protocol. Concerning direct administration systems, cotton tissue generally supports the diffusion in other highly practical methods. Despite preserving from essential oil decomposition, cotton does not promote aroma diffusion into the air [19]. This procedure has the advantage of avoiding volatile components' dispersion, but it does not guarantee blindness toward procedure; moreover, a preliminary sensibility test is recommended to avoid skin reactions. In fact, Kiecolt-Glaser and colleagues [31] considered their experimental system (soaked cotton ball with a fixed volume of EOs tapped between nose and upper-lip) better than environmental room inhalation as it guarantees more continuous and homogeneous exposure. Heuberger \& Ilmberger [27], Saiorwan et al. [32] and Kim et al. [34] administered EO using a face mask. In particular, Heuberger \& Ilmberger [27] applied to subjects a surgical mask coated with lavender EO; Saiorwan and colleagues [32] mixed volatile components of EO with oxygen through a pump connected to an oxygen mask; Kim and colleagues [34] applied a cotton swab soaked with EO to an oxygen mask. These methods allow the maintenance of $\mathrm{EO}$ quality preventing the environmental dispersion and possible contamination with other indoor volatiles. However, these advantages did not lead to subject blindness with respect to aroma diffusion. Therefore, direct and indirect EO diffusion systems can be considered as two complementary administration methods: on the one hand, if direct administration systems prevent from EO dispersion but may manipulate subjects expectancy toward the procedure, on the other hand, indirect administration systems guarantee subjects blindness but preclude EO components integrity. Expectancy effect manipulation induced by EO diffusion method will be more deeply discussed in the next paragraph. 


\subsection{Lavender EO and cognition}

As regards the effects of lavender EO inhalation on attention, the most frequent result emerging from the studies was reaction times decrease during sustained attention tasks $[33,35,36]$. These findings, together with less pronounced attentional blink found by Colzato and colleagues [29], suggest that lavender might induce a temporally distributed attentional state which positively influence performance in sustained attention tasks, at the expense of focused attentional state, as shown in Sellaro and colleagues' study [30]: in fact, lavender produced a more pronounced Simon effect, which is elicited by a task implying conflict monitoring abilities [42].

However, Chamine and colleagues [26] showed that faster reaction times in a vigilance task were induced by expectancy effect, since high expectations led to cognitive enhancement, regardless of intrinsic properties of lavender. These results are coherent with Heuberger and Ilmberger [27] study, which found that increase of performance speed was correlated with subjective ratings of odor valence. Therefore, these studies suggest that priming and judgement of the odor value were responsible for cognitive enhancement instead of lavender EO itself.

The aforementioned findings might be related to the arousal outcomes. The experimental procedures of these studies implied a stress-inducing situation leading to arousal increase, which led to performance detriment and which was found to be reversed by lavender inhalation. Indeed, Saiorwan and colleagues [32] found decrease in autonomic parameters and increase of band power in alpha, beta and theta waves during the lavender inhalation, indicating arousal decrease. These findings are corroborated by one EEG study which reported increased alpha wave after lavender inhalation, suggesting a relaxing effect [48]. Moreover, Diego and colleagues [47] showed a positive correlation between lavender EO inhalation and beta power increase, indicating arousal decrease; in the same study, arousal decline allowed the subjects to better perform sustained attention tasks. It is worth noting that Diego and colleagues [47] and Park and colleagues [48] also studied cognitive effects of lavender EO, however these studies were not included in this systematic review because were based on a within-subject design. Moreover, beta power is reported to be also related to less pronounced attentional blink [49] and this supports the hypothesis of a possible causal relation between lavender inhalation, arousal decrease and higher tonic attention performance. Since according to the Yerkes-Dodson law [50] cognitive performance is known to be optimal when arousal level is not too high and, if that range is surpassed, performance may decrease; thus, lavender EO might have decreased arousal to achieve more optimal levels in sustained attention tasks.

As regards the few and puzzling results about working memory, its improvement reported by Chamine and colleagues [26] might be explained by Yerkes-Dodson law [50], as well. Indeed, arousal decline induced by lavender can be considered as a cognitive substrate which promotes the deployment of higher cognitive functions, including working memory, whose structural and functional correlates partly overlap with those ones of attention [51]. However, Moss and colleagues [28] did not confirm the previous findings, reporting working memory decline after lavender EO inhalation. This incoherence might reflect the use of different neuropsychological tools to assess working memory: Chamine and colleagues [26] employed WAIS-III backward digit span [40], while Moss and colleagues used Cognitive Drug Research (CDR) battery [43]. It is well stated that a certain degree of task impurity can affect neuropsychological tasks. In this context, working memory subscale of CDR battery might be more specific for working memory assessment than WAIS-III backward digit span.

The previously mentioned results suggest potential central neural correlates of odor-induced effects. Focusing on lavender EO mechanism of action, the principal component of lavender is the acyclic monoterpene linalool, which has been shown to have GABA A receptors as main targets [52]. In fact, volatile components are reported to bind to odorant receptors at the olfactory epithelium [53]. Harada and colleagues [54] confirmed that linalool effect was mediated by GABA, since it was antagonized by flumazenil. Since GABAergic circuit has been found to actively modulate arousal in locus coeruleus [55], it is possible to assume that lavender EO might induce arousal decrease acting through GABAergic pathway. Moreover, this effect was not observed in anosmic mice, indicating that it was triggered by olfactory input evoked by linalool [54].

We can speculate that arousal decrement might be related to top-down processes: since orbitofrontal cortex receives projections from piriform cortex [56] and, together with anterior cingulate cortex, represents the only cortical structure to send efferent projections to locus coeruleus [57], arousal decrease might be induced by the modulation of locus coeruleus GABAergic neurons exerted by orbitofrontal cortex. In fact, neocortex is thought to be the main target of the 
sedative effects of volatile anesthetics, since its neurons are more sensitive to these chemical compounds [58]. However, other possible neurobiologic mechanisms of action of lavender volatile components are not to be excluded: since some evidence reported linalool to also act through serotonergic pathway [59], its effect could be ascribable to raphe nucleus activation by olfactory input from olfactory bulb [60], and subsequent bottom-up process to cortical areas [61].

However, since only one study [26] included placebo condition, a placebo effect or an overlapping between lavender EO mechanism of action and placebo effect are not to be definitely ruled out. Indeed, Masaoka and colleagues [62] reported a decrease in pain perception induced by expectancy of the analgesic effects of lavender; as compared to control group and subjects inhaling aroma without knowing it, this effect was more prominent in subjects who were informed they were inhaling an analgesic aroma and actually inhaled lavender EO, but it was also present among subjects who believed they were inhaling an analgesic EO but received no treatment. This study was not included in the systematic review because it investigated a psychological domain that does not concern the present study. Similarly, since placebo effect derives from subjective interpretation of context information and most part of medical treatments benefit is caused by the brain's response to the treatment context [63], visible EO diffusion devices might act as contextual cues, inducing an expectancy effect in subjects.

\subsection{Limitations and Future Perspectives}

Among the revised studies, several issues arise from a wide and unclear variety of terms used for the description of the administered EO. This fact has an important impact on the evaluation of efficacy of the inhalation protocol. The selection of lab-scale distilled EOs, commercial lavender EOs and marker composition should be based on the relative certificate of analysis in order to provide information linked to supplier and transformation process (plat raw material, extraction, carrier type, ratio dilution, process, lot number). Due to the frequent market adulteration for many plant derivatives, it is not possible to assume that any sold EO has been checked and has passed quality requirements. In fact, botanical authenticity and certification results are crucial to guarantee the subject's safety to better understand the relationship between $\mathrm{EO}$ volatile components and odor-induced effects on cognitive functions. In addition, batch-to-batch quali-quantitative chemical analysis should be required in each intervention session in order to check the chemical stability of the tested EO sample before its administration. Furthermore, the carrier oil used in the aromatic sample preparation should be described to study the stability and the correct EO posology in each exposure experimental session.

Regarding diffusion systems, EO stability should be guaranteed under controlled temperature and sample oxygen exposure in order to avoid any sort of EO degradation. Considering the direct or indirect administration systems during the exposure to EO, an oxygen mask with controlled EO flow and soaked cotton pad could be considered the most effective method to reduce this problem as compared to room diffusion through candles or electric systems. However, the lab-scale oxygen mask is generally described without any information about the operative flow. In this case, it is extremely important to focus on the composition of $\mathrm{EO}$ and the suitability carrier vehicle to adjust the right posology but also to avoid any allergic or undesirable reaction. For this reason, in the case of facial masks, more than in other types of diffusion systems, different doses of lavender EO should be administered in order to shed light on the minimum quantity needed to induce an effect on cognition. Although facial masks might guarantee a better controlled EO flow in administration protocols compared to environmental diffusion, its alleged placebo effect on the subject is not to be neglected in the evaluation of lavender inhalation effects.

Concerning cognitive enhancement effects, the experimental heterogeneity of the selected studies did not allow conducting a meta-analysis investigation. However, in light of the foregoing evidence, aromatherapy can be considered a practical and non-invasive method to enhance cognitive functions, for example to prevent serious consequences of work-related lapses of cognitive performance. Stronger conclusions can be obtained with the adoption of more specific and accurate experimental protocols for EO inhalation. In particular, the introduction of appropriate placebo conditions is required to better understand the underlying mechanisms of EO inhalation. To disentangle this issue, a placebo group with inert odor administration should be included and biasing contextual cues such as diffusion devices should be controlled. In this context, to avoid subject biasing and simultaneously preventing EO dispersion, treatment administration in delimited areas such as cubicles and the employment of a hidden diffusion device with a flowmeter should be considered. 


\section{Conclusions}

From this systematic review it was not possible to determine whether different EO components ratios have different impacts on cognitive function modulation, although cognitive enhancement induced by lavender EO inhalation was observed. However, the employment of different diffusion devices might have contaminated the pure effect of EO on cognition, due to EO degradation in indirect systems and expectancy effect induced by subjects' awareness of aroma inhalation in direct systems. Nevertheless, despite these biasing variables, lavender EO inhalation was shown to produce a decrease of arousal level and an improvement of sustained component of attention. Robust conclusion as regards memory domain cannot be stated, since only two studies investigated this function, reporting opposite results.

\section{References}

1. Ernst, E. The role of complementary and alternative medicine. BMJ 2000, 321(7269), 1133-5.

2. Hoffmann, D. Medical Herbalism; Healing Arts Press: Rochester, VT, USA, 2003.

3. Petersen, D. Aromatherapy: Characteristics of the market. In Proceedings of the 2013 International Federation of Essential Oils and Aroma Trades Conference, San Francisco, CA, September 2013.

4. Miller, L.; Miller, B. Ayurveda and Aromatherapy; Lotus Press: Twin Lakes, WI, USA, 1999.

5. Thomas, D.V. Aromatherapy: mythical, magical, or medicinal? Holistic Nursing Practice 2002, 16(5), 8-16.

6. Pharmacopée Française X Édition (Ph.Fr.X.). 1983, 1990. Moulins-les-Metz: Maisonneuve S.A.

7. Deutsches Arzneibuch, edition 1997 (DAB 1997).

8. European Pharmacopoeia (Ph. Eur.) 10th Edition.

9. WHO (2018), The International Pharmacopoeia (8th ed.).

10. EMA, HMPC. Community herbal monograph on. Lavandula angustifolia, 2012, 1-5.

11. Huet, R. Xème réunion du Comité Technique ISO/TC 54 "huiles essentielles". Fruits 1973, 28(1), 65-66.

12. Lawrence, B.M. Progress in EOs, lavender oils. Perfumer and Flavorist 1993, 18(1), 58-61.

13. Baser, K.H.C.; Özek, T.; Konakchiev, A. Enantiomeric distribution of linalool, linalyl acetate and camphor in Bulgarian lavender oil. Journal of EO Research 2011, 17, 135-136.

14. Maštovská, K.; Lehotay, S.J. Practical approaches to fast gas chromatography-mass spectrometry. Journal of Chromatography A 2003, 1000(1-2), 153-180.

15. Donelli, D.; Antonelli, M.; Bellinazzi, C.; Gensini, G.F.; Firenzuoli, F. Effects of lavender on anxiety: A systematic review and meta-analysis. Phytomedicine 2019, 65, 153099.

16. Zelano, C.; Jiang, H.; Zhou, G.; Arora, N.; Schuele, S.; Rosenow, J.; Gottfried, J.A. Nasal Respiration Entrains Human Limbic Oscillations and Modulates Cognitive Function. The Journal of Neuroscience 2016, 36(49), 12448-12468.

17. Cohen, Y.; Putrino, D.; Wilson, D.A. Dynamic cortical lateralization during olfactory discrimination learning. J Physiol 2015, 593(7), 1701-14.

18. Umezu, T.; Nagano, K.; Ito, H.; Kosakai, K.; Sakaniwa, M.; Morita, M. Anticonflict effects of lavender oil and identification of its active constituents. Pharmacol Biochem Behav 2006, 85(4), 713-21.

19. Halligudi, N.; Al Ojaili, M. The Science and Art of Aromatherapy: A Brief Review. Journal of Biomedical and Pharmaceutical Research 2013, 2(2), 06-14.

20. Moher, D.; Liberati, A.; Tetzlaff, J.; Altman, D.G. Preferred reporting items for systematic reviews and meta-analyses: the PRISMA statement. Annals of internal medicine 2009, 151(4), 264-269.

21. https://www.crd.york.ac.uk/prospero/

22. da Costa Santos, C.M.; de Mattos Pimenta, C.A.; Nombre, M.R. The PICO strategy for the research question construction and evidence search. Revista Latino-Americana de Enfermagem 2007, 15(3), 508-511.

23. Poldrack, R. (2011). Cognitive Atlas. http://www.cognitiveatlas.org/.

24. MENDELEY SUPPORT TEAM. (2011). Getting Started with Mendeley. Mendeley Desktop. London: Mendeley Ltd. Retrieved from http://www.mendeley.com.

25. Wells, G.A.; Shea, B.; O'Connell, D.A.; Peterson, J.; Welch, V.; Losos, M.; Tugwell, P. The Newcastle-Ottawa Scale (NOS) for assessing the quality of nonrandomized studies in meta-analyses. 2000.

26. Chamine, I.; Oken, B.S. Aroma Effects on Physiologic and Cognitive Function Following Acute Stress: A Mechanism Investigation. The Journal of Alternative and Complementary Medicine 2016, 22(9), 713-721.

27. Heuberger, E.; Ilmberger, J. The influence of essential oils on human vigilance. Natural Production Communications 2010, 5(9), 1441-1446. 
28. Moss, M.; Cook, J.; Wesnes, K.; Duckett, P. Aromas of rosemary and lavender EOs differentially affect cognition and mood in healthy adults. International Journal of Neuroscience 2003, 113, 15-38.

29. Colzato, L.S.; Sellaro, R.; Paccani, C.R.; Hommel, B. Attentional control in the attentional blink is modulated by odor. Atten Percept Psychophys 2014, 76, 1510-1515.

30. Sellaro, R.; Hommel, B.; Rossi Paccani, C.; Colzato, L.S. With peppermints you're not my prince: Aroma modulates self-other integration. Atten Percept Psychophys 2015, 77, 2817-2825.

31. Kiecolt-Glaser, J.K., Graham, J.E., Malarkey, W.B., Porter, K., Lemeshow, S., Glaser, R. (2008). Olfactory influences on mood and autonomic, endocrine, and immune function. Psychoneuroendocrinology, 33, 328-339.

32. Saiorwan, W.; Siripornpanich, V.; Piriyapunyaporn, T.; Hongratanaworakit, T.; Kotchabhakdi, N.; Ruangrungsi, N. The Effects of Lavender Oil Inhalation on Emotional States, Autonomic Nervous System, and Brain Electrical Activity. Journal of the Medical Association of Thailand 2012, 95(4), 598-606.

33. Shimizu, K.; Gyokusen, M.; Kitamura, S.; Kawabe, T.; Kozaki, T.; Ishibashi, K.; Izumi, R.; Mizunoya, W.; Ohnuki, K.; Kondo, R. Essential oil of lavender inhibited the decreased attention during a long-term task in humans. Biosci Biotechnol Biochem 2008, 72(7), 1944-7.

34. Kim, S.; Kim, H.-J.; Yeo, J.-S.; Hong, S.-J.; Lee, J.-M.; Jeon, Y. The Effect of Lavender Oil on Stress, Bispectral Index Values, and Needle Insertion Pain in Volunteers. The Journal of Alternative and Complementary Medicine 2011, 17(9), 823-826.

35. Sakamoto, R.; Minoura, K.; Usui, A.; Ishizuka, Y.; Kanba, S. Effectiveness of Aroma on Work Efficiency: Lavender Aroma during Recesses Prevents Deterioration of Work Performance. Chemical Senses 2005, 30, 683-691.

36. Yue, Z.; Gao, T.; Chen, L.; Wu, J. Odors Bias Time Perception in Visual and Auditory Modalities. Front Psychol $2016,7,535$.

37. Liu, J.; Singh, H.; White, P.F. Electroencephalogram bispectral analysis predicts the depth of midazolam-induced sedation. The Journal of the American Society of Anesthesiologists 1996, 84(1), 64-69.

38. Fassoulaki, A.; Paraskeva, A.; Patris, K.; Pourgiezi, T.; Kostopanagiotou, G. Pressure applied on the extra 1 acupuncture point reduces bispectral index values and stress in volunteers. Anesthesia E Analgesia 2003, 96(3), 885-890.

39. Stroop, J.R. Studies of interference in serial verbal reactions. Journal of Experimental Psychology 1935, 18(6), $643-662$.

40. Wechsler, D. WAIS-III Administration and Scoring Manual. Psychological Corp.: San Antonio, TX, USA, 1997.

41. Potter, M.C. Meaning in visual search. Science 1975, 187(4180), 965-6.

42. Simon, J.R.; Rudell, A.P. Auditory S-R compatibility: The effect of an irrelevant cue on information processing. Journal of Applied Psychology 1967, 51, 300-304.

43. Wesnes, K.A.; Wand, T.; Ayre, G.; Pincock, C. Validity and utility of the cognitive drug research (CDR) computerized assessment system: A review following fifteen years of usage. European Neuropsychopharmacology 1999, 9(sup. 5), S368.

44. Akira, N. Physiological background of aromatherapy. Japanese Soc Aromatherapy 2007, 6, 13-21.

45. Cavanagh, H.M.A.; Wilkinson, J.M. Biological activities of lavender EO. Phytother. Res. 2002, 16, 301-308.

46. Degel, J.; Koster, E. P. Odors: implicit memory and performance. Chem. Senses 1999, 24, 317-325.

47. Diego, M.A.; Jones, N.A.; Field, T.; Hernandez-Reif, M.; Schanberg, S.; Kuhn, C.; McAdam, V.; Galamaga, R.; Galamaga, M. Aromatherapy positively affects mood, EEG patterns of alertness and math computations. International Journal of Neuroscience 1998, 96(3-4), 217-24.

48. Park, K.H.; Kim, H.J.; Oh, B.; Seo, M.; Lee, E.; Ha, J. Evaluation of human electroencephalogram change for sensory effects of fragrance. Skin Res Technol 2019, 25(4), 526-531.

49. Gross, J.; Schmitz, F.; Schnitzler, I.; Kessler, K.; Shapiro, K.; Hommel, B.; Schnitzler, A. Modulation of long-range neural synchrony reflects temporal limitations of visual attention in humans. Proc Natl Acad Sci 2004, 101(35), 13050-5.

50. Yerkes, R.M.; Dodson, J.D. The relation of strength of stimulus to rapidity of habit-formation. Journal of Comparative Neurology and Psychology 1908, 18, 459-482.

51. Petersen, S.E.; Posner, M.I. The attention system of the human brain: 20 years after. Annual Review of Neuroscience 2012, 35, 73-89.

52. Hossain, S.J.; Hamamoto, K.; Aoshima, H.; Hara, Y. Effects of tea components on the response of GABA(A) receptors expressed in Xenopus Oocytes. Journal of Agriculture and Food Chemistry 2002, 50, 3954-3960.

53. Buck, L.B. Receptor diversity and spatial patterning in the mammalian olfactory system. Ciba Foundation Symposium 1993, 179, 51-64.

54. Harada, H.; Kashiwadani, H.; Kanmura, Y.; Kuwaki, T. Linalool Odor-Induced Anxiolytic Effects in Mice. Front Behav Neurosci 2018, 12, 241

55. Breton-Provencher, V.; Sur, M. Active control of arousal by a locus coeruleus GABAergic circuit. Nature neuroscience 2019, 22(2), 218-228.

56. Plailly, J.; Howard, J.D.; Gitelman, D.R.; Gottfried, J.A. Attention to odor modulates thalamocortical connectivity in the human brain. J Neurosci 2008, 28(20), 5257-67.

57. Aston-Jones, G.; Cohen, J. An Integrative Theory of Locus Coeruleus-Norepinephrine Function: Adaptive Gain and Optimal Performance. Annual Review of Neuroscience 2005, 28, 403-50.

58. Hentschke, H.; Schwarz, C.; Antkowiak, B. Neocortex is the major target of sedative concentrations of volatile anaesthetics: strong depression of firing rates and increase of GABA A receptor mediated inhibition. Eur J Neurosci 2005, 21, 93-102.

59. Chioca, L.R.; Ferro, M.M.; Baretta, I.P.; Oliveira, S.M.; Silva, C.R.; Ferreira, J.; Losso, E.M.; Andreatini, R. Anxiolytic-like effect of lavender essential oil inhalation in mice: participation of serotonergic but not GABA A/benzodiazepine neurotransmission. $J$ Ethnopharmacol 2013, 147(2), 412-8.

60. Miro, X.; Perez-Torres, S.; Palacios, J.M.; Puigdomènech, P.; Mengod, G. Differential distribution of cAMP-specific phosphodiesterase 7A mRNA in rat brain and peripheral organs. Synapse 2001, 40, 201-214. 
61. Kandel, E.; Schwartz, J.H. Principles of Neural Sciences, Vol.2; Elsevier: New York, 1985.

62. Masaoka, Y.; Takayama, M.; Yajima, H.; Kawase, A.; Takakura, N.; Homma, I. Analgesia Is Enhanced by Providing Information regarding Good Outcomes Associated with an Odor: Placebo Effects in Aromatherapy? Evid Based Complement Alternat Med 2013, 921802.

63. Wager, T.D.; Atlas, L.Y. The neuroscience of placebo effects: connecting context, learning and health. Nature Reviews Neuroscience 2015, 16(7), 403-18. 